\title{
A Novel Joint Localization Method for Acoustic Emission Source Based on Time Difference of Arrival and Beamforming
}

\author{
Xiaoran Wang, Xiandong Liu, Tian He *, Junfei Tai and Yingchun Shan \\ School of Transportation Science and Engineering, Beihang University, Beijing 100191, China; \\ wangxiaoran@buaa.edu.cn (X.W.); liuxiandong@buaa.edu.cn (X.L.); taijunfei@buaa.edu.cn (J.T.); \\ shanych@buaa.edu.cn (Y.S.) \\ * Correspondence: hetian@buaa.edu.cn; Tel.: +86-131-4128-1907
}

Received: 18 October 2020; Accepted: 9 November 2020; Published: 13 November 2020

\begin{abstract}
Time difference of arrival (TDOA) method and beamforming method are often individually utilized in the localization of acoustic emission source of a plate. Based on the performances of the two methods, a novel joint localization method for acoustic emission source is proposed in this paper. Firstly, the performances of TDOA method and beamforming method are studied based on the simulation signals. Then, aiming at the advantages and disadvantages of these two methods, a joint localization method is proposed. Finally, the performances of joint localization method are verified by simulation and experiment. Both simulation and experimental results show that the accuracy of the joint localization method is improved, and the calculation amount is greatly reduced in comparison to the TDOA method and the beamforming method.
\end{abstract}

Keywords: time difference of arrival; beamforming; joint localization; acoustic emission

\section{Introduction}

Acoustic emission (AE) is defined as a phenomenon whereby a transient elastic wave is generated by the rapid release of energy from a localized source of damage [1]. Various types of AE sources, such as fiber breakage [2], fatigue cracks [3], rubbing [4], and impact of foreign objects [5] can generate the propagation of $\mathrm{AE}$ waves. The process of locating the source of these acoustic waves, by recording the propagating acoustic signals from various sensors and properly analyzing them, is commonly known as the AE localization technique. It is an important step for structural health monitoring (SHM) [6]. The AE localization technique is one of the key issues in $\mathrm{AE}$ technology research. The accuracy of the localization reflects the degree of compliance between the detection location of the AE source and real location.

Many scientists have conducted a mass of research on the source localization of $\mathrm{AE}$, and have proposed many efficient localization methods. Kundu [6] reviewed the research status of AE source localization research technology. Tobias [7] is a pioneer in the study of AE source localization in isotropic materials. The triangulation method proposed by Tobias is the most commonly used method for isotropic materials, which determines the location of the AE source based on the time difference of arrival (TDOA) between the AE waves reaching different sensors. But only when the precise wave velocity of elastic wave propagation in the material is obtained can an accurate location result be obtained. In response to this limitation, Kundu et al. [5] improved the triangulation method and proposed an AE source localization method suitable for isotropic materials without wave velocity. However, the localization accuracy of the TDOA method is affected by noise, dispersion effect, energy attenuation, and other factors during the propagation process [8]. Moreover, the application of 
TDOA method in two-dimensional or three-dimensional large and medium-sized structures generally requires the sensors to be sparsely arranged. In response to this situation, McLaskey et al. [9] carried out non-destructive testing research based on the beamforming method to achieve the location of $\mathrm{AE}$ sources for structural damage in the field of civil engineering. He et al. [10] introduced the near-field beamforming method into the processing of AE signals in thin-plate structures, and studied a new method of arranging a small number of sensors in small areas to realize the localization of $\mathrm{AE}$ sources.

In order to locate the acoustic emission source in an anisotropic material, Nakatani et al. [11,12] extended the beamforming method into anisotropic materials. But it is necessary to measure the propagation velocity of AE waves in different directions in anisotropic materials. Kundu et al. [5,13,14], Hajzargerbashi et al. [15], and Koabaz et al. [16] improved the TDOA method and successfully applied it to AE source localization of anisotropic materials. The localization accuracy of these methods depends on the measured wave velocity, and the relevant properties of the material need to be obtained in advance. In order to overcome the influence of wave velocity on the localization accuracy of $\mathrm{AE}$ sources in anisotropic materials, Kundu et al. $[17,18]$ proposed an AE source localization method that does not require wave velocity. Only six acoustic emission sensors are needed to locate AE source in an anisotropic plate.

In summary, the localization research of AE source mainly includes the TDOA method and the beamforming method. These methods can be applied to actual engineering structures. For example, fuselages of aviation structures [19], aircraft engines [20], and vehicle welding structures [21]. Although the TDOA method is mature and easy to use, its localization effect is highly dependent on the accuracy of the obtained TDOA. The beamforming method based on the delay-and-sum algorithm is accurate in localization. However, it is necessary to divide the plate into meshes according to the required accuracy and then perform scanning calculations. This results in a larger calculation cost and a lower localization speed.

Based on the localization performances of TDOA method and beamforming method, this paper proposes a joint localization method. The proposed method not only improves the accuracy of the TDOA method, but also greatly reduces the localization calculation coat of the beamforming method. The organization of this paper is as follows. The principle of TDOA method and beamforming method are introduced in Section 2. Section 3 analyses the performances of TDOA method and beamforming method based on simulated signals. Section 4 proposes the joint localization method. Section 5 carries out simulation and experimental verification. Finally, the conclusions are drawn in Section 6.

\section{Principle of TDOA Method and Beamforming Method}

\subsection{TDOA Method for Four Sensors}

Four sensors linear arrays are used for locating here. The sensor arrangement is shown in Figure 1. Four sensors (1), (2), (3), and (4) in Figure 1) arranged adjacent to each other at equal intervals and P is the location of $\mathrm{AE}$ source. Suppose the distance between two adjacent sensors is $\mathrm{d}$ and the speed of AE wave is $v$. The time that AE signal generated by AE source $P(x, y)$ reaches the sensor (1) is $t_{1}$. The time differences of arrival between signal arrival times at sensor (2), (3), (4), and sensor (1) are $\Delta t_{2}$, $\Delta t_{3}$, and $\Delta t_{4}$, respectively. AE source coordinates satisfy the following relationships

$$
\left\{\begin{array}{l}
x^{2}+y^{2}=v^{2} t_{1}^{2} \\
(x-d)^{2}+y^{2}=v^{2}\left(\Delta t_{2}+t_{1}\right)^{2} \\
(x-2 d)^{2}+y^{2}=v^{2}\left(\Delta t_{3}+t_{1}\right)^{2} \\
(x-3 d)^{2}+y^{2}=v^{2}\left(\Delta t_{4}+t_{1}\right)^{2}
\end{array}\right.
$$




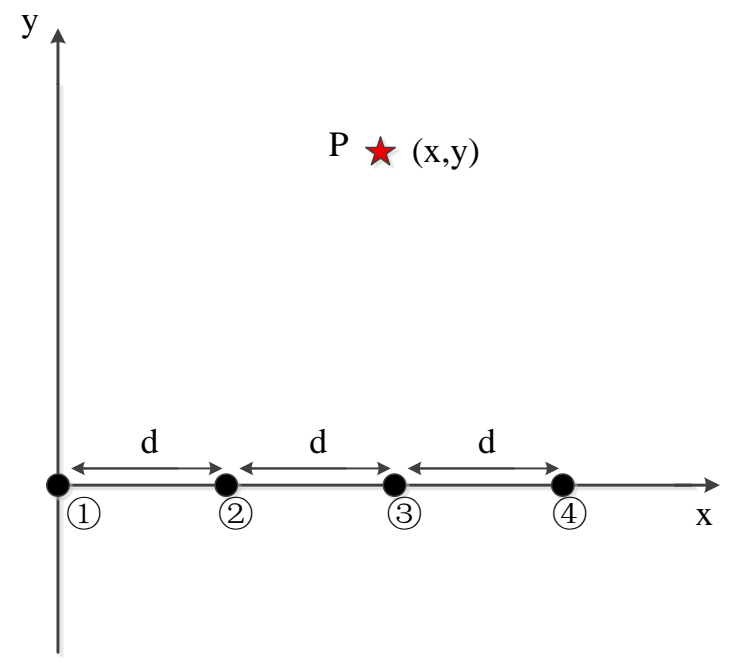

Figure 1. Linear array of four sensors.

By subtracting formula 1 from formula 2, 3, and 4 in Equation (1), we can get that

$$
\left\{\begin{array}{l}
-2 x d+d^{2}=v^{2}\left(2 t_{1} \Delta t_{2}+\Delta t_{2}^{2}\right) \\
-4 x d+4 d^{2}=v^{2}\left(2 t_{1} \Delta t_{3}+\Delta t_{3}^{2}\right) \\
-6 x d+9 d^{2}=v^{2}\left(2 t_{1} \Delta t_{4}+\Delta t_{4}^{2}\right)
\end{array}\right.
$$

Then, we can get

$$
t_{1}=\frac{3 \Delta t_{2}^{2}-3 \Delta t_{3}^{2}+\Delta t_{4}^{2}}{-6 \Delta t_{2}+6 \Delta t_{3}-2 \Delta t_{4}}
$$

Substituting Equation (3) into formula 1 and 2 in Equation (2), respectively, we can get unitary quadratic equations with $x$ and $v^{2}$ as unknowns. Solving the equations, we can get

$$
x=\frac{d\left(8 t_{1} \Delta t_{2}+4 \Delta t_{2}^{2}-2 t_{1} \Delta t_{3}-\Delta t_{3}^{2}\right)}{8 t_{1} \Delta t_{2}+4 \Delta t_{2}^{2}-4 t_{1} \Delta t_{3}-2 \Delta t_{3}{ }^{2}}
$$

Dividing formula 1 by formula 2 in Equation (1), we can get

$$
\frac{x^{2}+y^{2}}{(x-d)^{2}+y^{2}}=\frac{t_{1}^{2}}{\left(\Delta t_{2}+t_{1}\right)^{2}}
$$

Solving Equation (5), we can get

$$
y=\sqrt{\frac{t_{1}^{2} d^{2}-2 x d t_{1}^{2}}{\Delta t_{2}^{2}+2 \Delta t_{2} t_{1}}-x^{2}}
$$

\subsection{Beamforming Method}

The beamforming method theoretically can identify the location of any AE source in the near-field region. When the array is focused to a point source at limited distance, the incident AE waves are spherical, as shown in Figure 2. Array output is calculated by [20]

$$
b(\vec{r}, t)=\frac{1}{M} \sum_{m=1}^{M} w_{m} x_{m}\left(t-\Delta_{m}(\vec{r})\right)
$$


where $b(\vec{r}, t)$ is the output of the array; $\vec{r}$ is the direction vector from the reference sensor to the focused point. The reference point may be arbitrary and it is the first sensor point on the left side in the Figure 2; $M$ is the number of sensors; $w_{m}$ the weighting coefficient for the channel of sensor $m$ (usually no modification, in this article, $w_{m} \equiv 1$ ) and $x_{m}(t)$ represents the signal acquired from the No. $m$ sensor; $\Delta_{m}(\vec{r})$ indicates the individual time delay of No. $m$ sensor to the reference point.

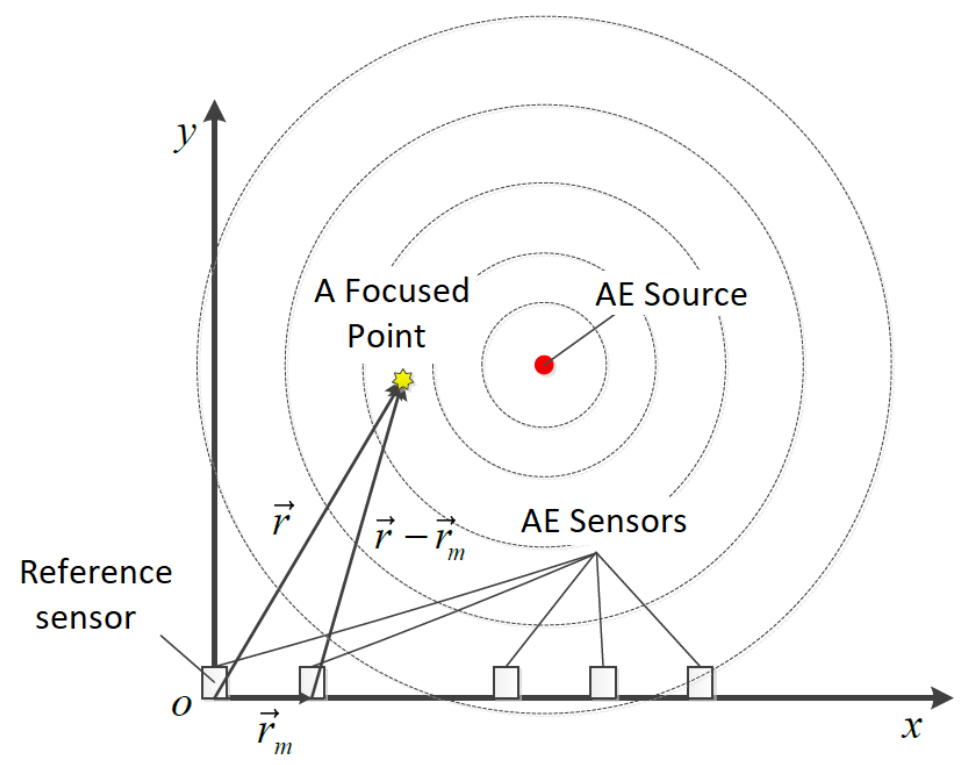

Figure 2. Schematic diagram of the principle of beamforming method.

By adjusting time delay $\Delta_{m}(\vec{r})$, the signals associated with the spherical waves, emitting from AE source focus, will be aligned in time before they are summed. As shown in Figure 2, $\Delta_{m}(\vec{r})$ can be obtained by

$$
\Delta_{m}(\vec{r})=\frac{|\vec{r}|-\left|\vec{r}-\vec{r}_{m}\right|}{c}
$$

where $\vec{r}_{m}$ the distance between reference point and No. $m$ sensor; $|\vec{r}|-\left|\vec{r}-\vec{r}_{m}\right|$ represents the difference between the distance from reference point to focus point and the distance from No. $m$ sensor to the focus point; $c$ is the propagation velocity of $\mathrm{AE}$ wave.

The output of the beamforming method is the integral of $b(\vec{r}, t)$ over time, which has the meaning of energy. If the focused point is the real source, the signals are aligned at the same wave front and the output of the beamforming is maximum. However, the signals cannot be aligned at the same wave front when the array of sensors is focused on other locations, and the output of the beamforming is not the maximum. The location of the maximum output energy represents the location of the AE source. When the beamforming method is used, every point on the structure is focused, and the output of the beamforming array at every point needs to be calculated. Once the location of the real AE source is focused on, the output of the sensor array reaches the maximum value. Thus, the location of the real AE source can be judged by comparing those outputs. Figure 3 shows the operational principle of the beamforming method. 


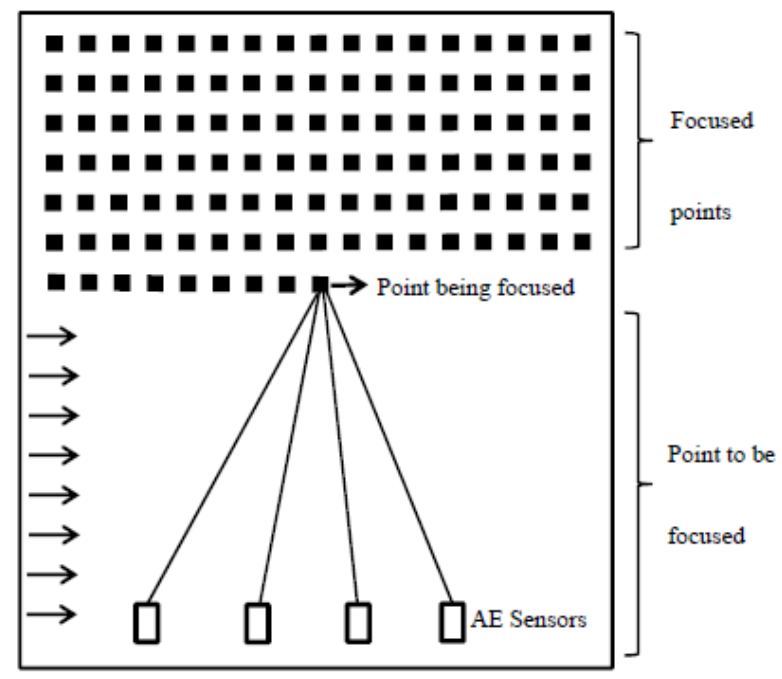

Figure 3. The operational principle of the beamforming method.

In operation, the localization accuracy can be determined according to actual needs. For example, on a $600 \mathrm{~mm}$ by $600 \mathrm{~mm}$ plate, if locating is performed with an accuracy of $10 \mathrm{~mm}, 3721(61 \times 61)$ points need to be scanned. If locating is performed with an accuracy of $1 \mathrm{~mm}, 361,201(601 \times 601)$ points need to be scanned. Obviously, the higher the localization accuracy, the more points need to be scanned. This means that the longer it takes to locate. Then the output of each point can be calculated. The point with the largest output value is the location of the acoustic emission source. Figure 4 shows the beamforming array output in a typical acoustic emission source location. At this time, normalization is performed according to the maximum output value. It can be seen from the Figure 4 that the location where the output is one is the acoustic emission source.

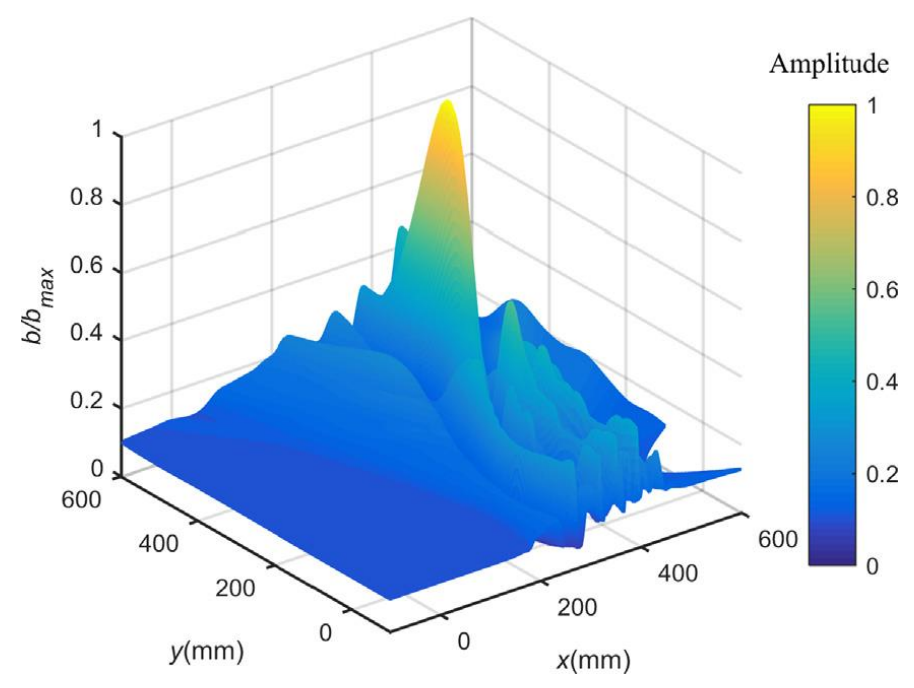

Figure 4. A typical energy output of beamforming method [22].

The beamforming method has high localization accuracy. However, the localization speed is very low since great quantities of points need to be scanned. The calculation speed is affected by the length of the calculation signal, the number of sensors, the localization accuracy (the higher the accuracy, the more points need to be scanned), and the size of the structure. Among these influencing factors, signal length, number of sensors, localization accuracy, and structural size are all rigid requirements, and it is difficult to change them for the speed of calculation. 


\section{Performance Analysis of TDOA Method and Beamforming Method Based on the} Simulated Signals

Since the finite element simulation signal is close to the actual AE signal and easy to operate, this paper uses the simulation signal to study the performance of TDOA method and beamforming method for localizing AE source.

\subsection{Simulation of $A E$ Source}

In this section, the commercial software Abaqus/Explicit is used to obtain the typical signals of the AE sources. In the simulation, the element type is C3D8R; the element size is $1 \mathrm{~mm}$; the time increment step is set to be $1 \times 10^{-8} \mathrm{~s}$; the sampling interval is $2 \times 10^{-7} \mathrm{~s}$; and the total simulation time is $3 \times 10^{-4} \mathrm{~s}$. The quadrupole force is used to simulate AE source. As shown in Figure 5a, the concentrated forces with $1 \mathrm{~N}$ magnitude are loaded on the four nodes, respectively. The time history of these forces, as triangular pulse form, is shown in Figure 5b. Here, the S0 wave data are selected for localization.
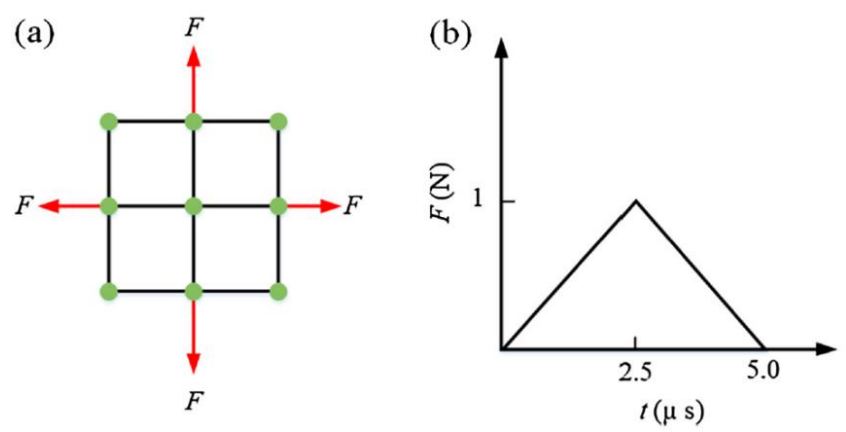

Figure 5. Excitation information: (a) The loading location; (b) The mechanical representation.

\subsection{Performance Analysis of TDOA Method}

AE simulation is carried out on steel plates with dimensions of $800 \mathrm{~mm}$ by $1000 \mathrm{~mm}$ by $5 \mathrm{~mm}$ and the location of the 15 sensors is shown in Figure 6. The spacing of each sensor is $50 \mathrm{~mm}$. The coordinates of the first sensor on the left are $(50 \mathrm{~mm}, 100 \mathrm{~mm})$. The location of AE source is set to \#1 (300 mm, $600 \mathrm{~mm}$ ) and \#2 (500 mm, $800 \mathrm{~mm})$. Material properties used in the finite element model are tabulated as Table 1.

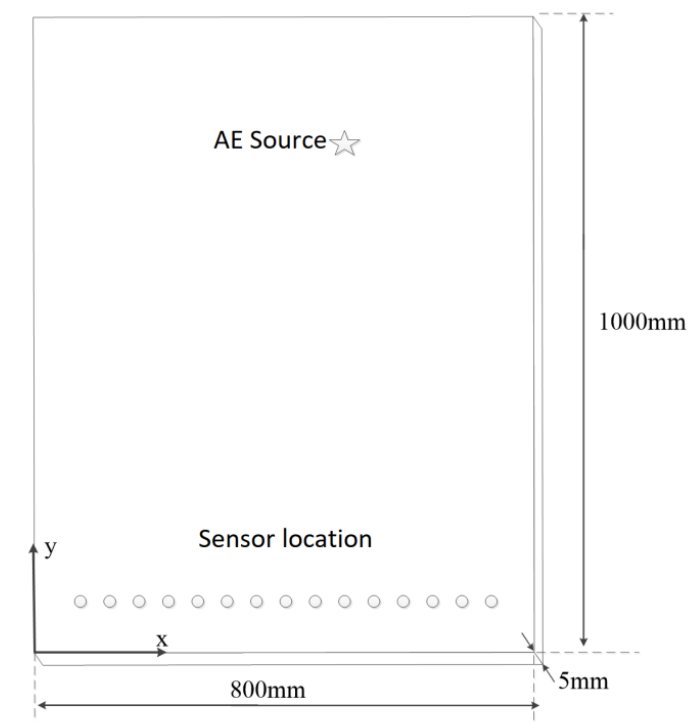

Figure 6. Steel plate simulation model. 
Table 1. Material properties.

\begin{tabular}{cccc}
\hline Parameters & $\boldsymbol{E}$ & $\boldsymbol{\rho}$ & $\boldsymbol{V}$ \\
\hline Value & 209 & 7800 & 0.3 \\
Units & $\mathrm{GPa}$ & $\mathrm{Kg} / \mathrm{m}^{3}$ & $/$ \\
\hline
\end{tabular}

The simulated signals received by the 15 sensors are extracted for locating. Based on the first sensor on the left, the TDOA locating of four sensors is carried out according to the sensor spacing of $50 \mathrm{~mm}, 100 \mathrm{~mm}, 150 \mathrm{~mm}$, and $200 \mathrm{~mm}$, respectively. The localization results are shown in Table 2 .

Table 2. Time difference of arrival (TDOA) locating of different sensor spacing.

\begin{tabular}{ccccc}
\hline $\begin{array}{c}\text { Sensor Spacing } \\
(\mathbf{m m})\end{array}$ & $\begin{array}{c}\text { Actual Location of \#1 } \\
(\mathbf{m m})\end{array}$ & $\begin{array}{c}\text { Localization } \\
\mathbf{( m m})\end{array}$ & $\begin{array}{c}\text { Actual Location of \#2 } \\
(\mathbf{m m})\end{array}$ & $\begin{array}{c}\text { Localization } \\
(\mathbf{m m})\end{array}$ \\
\hline 50 & $(300,600)$ & $(286,281)$ & $(500,800)$ & $(650,762)$ \\
100 & $(300,600)$ & $(300,334)$ & $(500,800)$ & $(534,1010)$ \\
150 & $(300,600)$ & $(301,381)$ & $(500,800)$ & $(495,757)$ \\
200 & $(300,600)$ & $(300,586)$ & $(500,800)$ & $(503,834)$ \\
\hline
\end{tabular}

It can be concluded from Table 2 that the x-coordinates error located by the TDOA method of the four sensors for the linear array is small. When the $\mathrm{x}$-coordinate of AE source is within the sensor array, the $\mathrm{x}$-coordinate of the localization point is very accurate, but the $y$-coordinate is generally not accurate.

The relationship between the localization of four sensors and the TDOA error is studied by using the TDOA of ideal wave. Set AE wave propagation speed in the plate to $5400 \mathrm{~m} / \mathrm{s}$. AE source locations are selected as $(350 \mathrm{~mm}, 600 \mathrm{~mm}),(550 \mathrm{~mm}, 600 \mathrm{~mm})$, and $(750 \mathrm{~mm}, 600 \mathrm{~mm})$. Sensor spacing is $200 \mathrm{~mm}$. The coordinates of number $1-4$ sensors are $(100 \mathrm{~mm}, 0 \mathrm{~mm}),(300 \mathrm{~mm}, 0 \mathrm{~mm}),(500 \mathrm{~mm}, 0 \mathrm{~mm})$, and $(700 \mathrm{~mm}, 0 \mathrm{~mm})$. Firstly, it is assumed that the TDOA between the times of AE wave arriving at number 2 and 3 sensors to that of number 1 sensor remains unchanged. The TDOA between number 4 sensor and number 1 sensor is changed to deviate from within $10 \%$ of the correct value and observe the localization result. Then, it is assumed that the TDOA between the times of AE wave arriving at number 2 and 4 sensors to that of number 1 sensor remain unchanged. The TDOA between number 3 sensor and number 1 sensor is changed to deviate from within $10 \%$ of the correct value. Finally, it is assumed that the TDOA between the times of AE wave arriving at number 3 and 4 sensors to that of number 1 sensor are unchanged. The TDOA between the number 2 sensor and number 1 sensor is changed, and the deviation is also within $10 \%$ of the correct value. The localization results are shown in Table 3. N.A. indicates that the localization result has an imaginary number.

It can be concluded from Table 3 that when the TDOA fluctuates within a certain range, the localization result of the $x$-coordinate does not change much, but the range of the $y$-coordinate of the localization varies greatly. Sometimes the localization result may be an imaginary number.

Table 3. Localization results of changing TODA.

\begin{tabular}{ccccc}
\hline $\begin{array}{c}\text { AE Source } \\
\text { Location }\end{array}$ & $\begin{array}{c}\text { Change in TODA } \\
(\%)\end{array}$ & $\begin{array}{c}\text { Change } \Delta t_{\mathbf{2}} \\
\text { Unchange } \Delta \boldsymbol{t}_{\mathbf{3}}, \Delta \boldsymbol{t}_{\mathbf{4}}\end{array}$ & $\begin{array}{c}\text { Change } \Delta \boldsymbol{t}_{\mathbf{3}} \\
\text { Unchange } \Delta \boldsymbol{t}_{\mathbf{2}}, \Delta \boldsymbol{t}_{\mathbf{4}}\end{array}$ & $\begin{array}{c}\text { Change } \Delta \boldsymbol{t}_{\mathbf{4}} \\
\text { Unchange } \Delta \boldsymbol{t}_{\mathbf{2}}, \Delta \boldsymbol{t}_{\mathbf{3}}\end{array}$ \\
\hline-10 & N.A. & $(345,310)$ & $(351,383)$ \\
& -8 & N.A. & $(346,340)$ & $(351,413)$ \\
& -6 & N.A. & $(347,377)$ & $(351,447)$ \\
& -4 & N.A. & $(348,426)$ & $(350,488)$ \\
$(350,600)$ & -2 & $(351,1020)$ & $(349,495)$ & $(350,538)$ \\
& $(349,456)$ & $(351,801)$ & $(350,683)$ \\
& 4 & $(348,376)$ & $(352,1529)$ & $(350,800)$ \\
& 6 & $(348,323)$ & N.A. & $(349,992)$ \\
& 10 & $(347,284)$ & N.A. & $(349,1400)$ \\
& $(347,253)$ & N.A. & $(349,5150)$ \\
\hline
\end{tabular}


Table 3. Cont.

\begin{tabular}{|c|c|c|c|c|}
\hline $\begin{array}{l}\text { AE Source } \\
\text { Location }\end{array}$ & $\begin{array}{c}\text { Change in TODA } \\
(\%)\end{array}$ & $\begin{array}{c}\text { Change } \Delta t_{2} \\
\text { Unchange } \Delta t_{3}, \Delta t_{4}\end{array}$ & $\begin{array}{c}\text { Change } \Delta t_{3} \\
\text { Unchange } \Delta t_{2}, \Delta t_{4}\end{array}$ & $\begin{array}{c}\text { Change } \Delta t_{4} \\
\text { Unchange } \Delta t_{2}, \Delta t_{3}\end{array}$ \\
\hline \multirow{10}{*}{$(550,600)$} & -10 & $(560,243)$ & N.A. & $(525,365)$ \\
\hline & -8 & $(558,280)$ & N.A. & $(530,396)$ \\
\hline & -6 & $(556,326)$ & N.A. & $(534,432)$ \\
\hline & -4 & $(554,386)$ & N.A. & $(539,476)$ \\
\hline & -2 & $(552,468)$ & $(554,1222)$ & $(544,530)$ \\
\hline & 2 & $(548,880)$ & $(547,429)$ & $(556,696)$ \\
\hline & 4 & $(546,4811)$ & $(544,338)$ & $(563,840)$ \\
\hline & 6 & N.A. & $(542,279)$ & $(570,1096)$ \\
\hline & 8 & N.A. & $(540,236)$ & $(578,1801)$ \\
\hline & 10 & N.A. & $(538,202)$ & N.A. \\
\hline \multirow{10}{*}{$(750,600)$} & -10 & $(660,112)$ & N.A. & $(638,299)$ \\
\hline & -8 & $(675,168)$ & N.A. & $(653,330)$ \\
\hline & -6 & $(691,233)$ & N.A. & $(670,369)$ \\
\hline & -4 & $(709,315)$ & N.A. & $(691,421)$ \\
\hline & -2 & $(728,427)$ & $(835,1702)$ & $(717,492)$ \\
\hline & 2 & $(774,940)$ & $(702,372)$ & $(795,784)$ \\
\hline & 4 & $(802,2844)$ & $(672,262)$ & $(861,1195)$ \\
\hline & 6 & N.A. & $(651,195)$ & $(965,4644)$ \\
\hline & 8 & N.A. & $(637,146)$ & N.A. \\
\hline & 10 & N.A. & $(626,108)$ & N.A. \\
\hline
\end{tabular}

\subsection{Performance Analysis of Beamforming Method}

The simulation signals received by the above 15 sensors are extracted. The accuracy of $10 \mathrm{~mm}$ is used for beamforming method (same below). The number of points to be scanned is $8181(101 \times 81)$, and the beamforming locating is performed, respectively, according to the sensor spacings of $50 \mathrm{~mm}$, $100 \mathrm{~mm}, 150, \mathrm{~mm}$ and $200 \mathrm{~mm}$. According to the method and operation steps introduced in Section 2.2, the localization results are shown in Table 4.

Table 4. Beamforming localization results.

\begin{tabular}{ccccc}
\hline $\begin{array}{c}\text { Sensor Spacing } \\
(\mathbf{m m})\end{array}$ & $\begin{array}{c}\text { Actual Location of \#1 } \\
(\mathbf{m m})\end{array}$ & $\begin{array}{c}\text { Localization } \\
(\mathbf{m m})\end{array}$ & $\begin{array}{c}\text { Actual Location of \#2 } \\
(\mathbf{m m})\end{array}$ & $\begin{array}{c}\text { Localization } \\
(\mathbf{m m})\end{array}$ \\
\hline 50 & $(300,600)$ & $(330,690)$ & $(500,800)$ & $(560,940)$ \\
100 & $(300,600)$ & $(300,610)$ & $(500,800)$ & $(490,800)$ \\
150 & $(300,600)$ & $(300,610)$ & $(500,800)$ & $(500,820)$ \\
200 & $(300,600)$ & $(300,610)$ & $(500,800)$ & $(500,820)$ \\
\hline
\end{tabular}

It can be seen from the localization results in Table 3 that the localization of the beamforming method is very accurate. As the distance between the sensors increases, the localization is more accurate. However, this method needs to divide the plate, and each divided point must be scanned and calculated, which increases the calculation cost.

\section{Joint Localization Method for TDOA and Beamforming}

From the above analysis, it can be known that if the four sensors are used for TDOA locating, the approximate location of AE source can be located. Then, the region located by the TDOA method is divided elaborately, and the beamforming method is used to locate AE source. This joint localization method is more accurate than the TDOA method, and also reduces the calculation cost in comparison to the beamforming method.

The specific steps are:

(1) As shown in Figure 7, four sensors are placed on the plate which is divided according to the midpoint of each two adjacent sensors. Then the plate can be divided into four regions: $\langle 1\rangle,\langle 2\rangle$, 
$<3>$, and $<4>$. The sensors (1)-(4) are used for TDOA locating. The $x$-coordinate of the AE source can be obtained.

(2) The localization based on the beamforming method is performed on the corresponding region according to the $\mathrm{x}$ coordinate. For example, if the $\mathrm{x}$-coordinate of the TDOA locating is in the region $\langle 1\rangle$, the region $<1>$ is subjected to the beamforming elaborate scanning for locating.

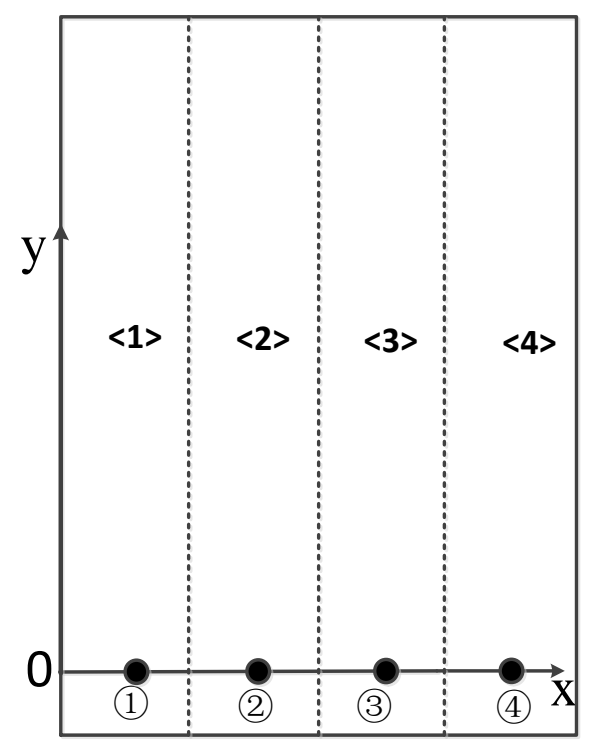

Figure 7. Schematic diagram of joint location method.

\section{Simulation and Experimental Verification}

In order to verify the effectiveness of the joint localization method proposed in this paper, the simulation is used to extract the signal for localization, and then the broken-lead experiment is performed on the steel plate.

\subsection{Simulation Verification}

AE simulation is carried out on steel plates with dimension of $800 \mathrm{~mm}$ by $700 \mathrm{~mm}$ by $5 \mathrm{~mm}$. The sensors are arranged as Figure 5 with a spacing of $200 \mathrm{~mm}$. The x-coordinates of AE source are set to $350 \mathrm{~mm}, 450 \mathrm{~mm}, 550 \mathrm{~mm}, 550 \mathrm{~mm}$, and $750 \mathrm{~mm}$, respectively, and the y-coordinates are set to $300 \mathrm{~mm}, 400 \mathrm{~mm}, 500 \mathrm{~mm}$, and $600 \mathrm{~mm}$, respectively. According to the method and operation steps introduced in Section 2.2, the localization results are shown in Table 5.

From Table 5, it can be concluded that after the x-coordinates of AE source are initially located by the TDOA method, the beamforming localization is carried out, and the localization results are more accurate. Due to the beamforming method scanning a quarter of the entire plate, the calculation cost of the localization is greatly reduced. The TDOA localization results in the Table 5 are also relatively accurate because the signal from the simulation data has no noise interference. There is a certain error in the actual TDOA. 
Table 5. Simulation localization results.

\begin{tabular}{ccccc}
\hline \multicolumn{2}{c}{ Actual Location } & $\begin{array}{c}\text { TDOA Localization } \\
\text { Result }(\mathbf{m m})\end{array}$ & $\begin{array}{c}\text { Beamforming } \\
\text { Scanning Region }\end{array}$ & $\begin{array}{c}\text { Beamforming } \\
\text { Localization Result (mm) }\end{array}$ \\
\hline $\mathbf{x}(\mathbf{m m})$ & $\mathbf{y}(\mathbf{m m})$ & $(350,614)$ & $<2>$ & $(350,610)$ \\
350 & $(450,614)$ & $<3>$ & $(450,610)$ \\
450 & $(550,608)$ & $<3>$ & $(550,610)$ \\
550 & $(549,579)$ & $<4>$ & $(650,620)$ \\
650 & $(647,571)$ & $<2>$ & $(750,630)$ \\
750 & $(350,486)$ & $<3>$ & $(350,510)$ \\
350 & $(450,486)$ & $<3>$ & $(450,510)$ \\
450 & $(550,508)$ & $<4>$ & $(550,510)$ \\
550 & $(650,522)$ & $<4>$ & $(650,520)$ \\
650 & $(746,470)$ & $<2>$ & $(750,530)$ \\
750 & & $(350,410)$ & $<3>$ & $(350,410)$ \\
350 & & $(450,410)$ & $<3>$ & $(450,410)$ \\
450 & $(550,404)$ & $<4>$ & $(550,410)$ \\
550 & & $(649,390)$ & $(650,420)$ \\
650 & & $(748,393)$ & $<4>$ & $(760,440)$ \\
750 & 400 & $(350,284)$ & $<3>$ & $(350,310)$ \\
350 & $(450,284)$ & $<3>$ & $(450,310)$ \\
450 & $(550,292)$ & $<4>$ & $(550,310)$ \\
550 & $(650,305)$ & $<4>$ & $(650,320)$ \\
650 & $(750,294)$ & & $(750,330)$ \\
750 & & &
\end{tabular}

\subsection{Experimental Verification}

In order to further verify the feasibility of joint localization method proposed in this paper, the broken-lead experiment is performed on the steel plate, as shown in Figure 8. It includes four $\mathrm{AE}$ sensors connected to the preamplifier to obtain the signal, and the data acquisition instrument connected to the PC to analyze and process the signal. The sensors are arranged in a row with coordinates $(150 \mathrm{~mm}, 0 \mathrm{~mm}),(270 \mathrm{~mm}, 0 \mathrm{~mm}),(390 \mathrm{~mm}, 0 \mathrm{~mm})$, and $(510 \mathrm{~mm}, 0 \mathrm{~mm})$, respectively. The sensor spacing is $120 \mathrm{~mm}$. The broken-lead experiments are performed at the location of AE sources shown in Table 6, and the signal received by the sensors are extracted. According to the method and operation steps introduced in Section 2.2, the localization results are shown in Table 6. N.A. indicates that the localization result has an imaginary number.

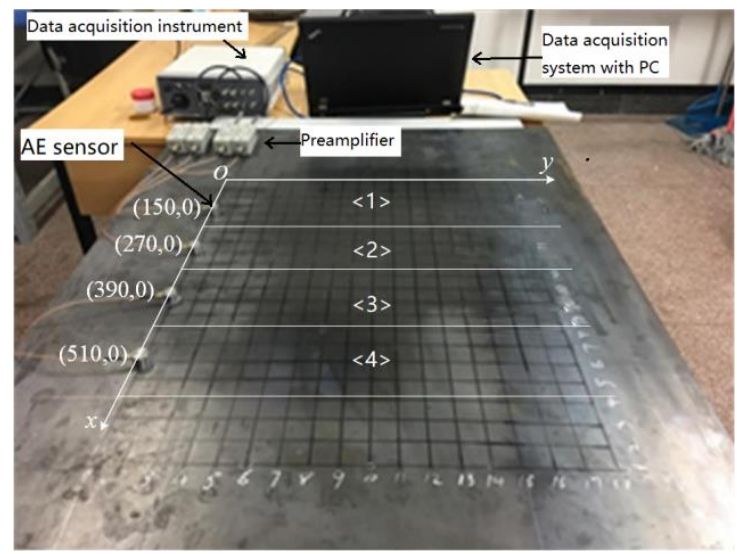

Figure 8. Experimental setup of acoustic emission (AE) detection. 
Table 6. Experimental localization results.

\begin{tabular}{|c|c|c|c|c|}
\hline \multicolumn{2}{|c|}{ Actual Location } & \multirow{2}{*}{$\begin{array}{l}\text { TDOA Localization } \\
\text { Result (mm) }\end{array}$} & \multirow{2}{*}{$\begin{array}{l}\text { Beamforming } \\
\text { Scanning Region }\end{array}$} & \multirow{2}{*}{$\begin{array}{c}\text { Beamforming } \\
\text { Localization Result (mm) }\end{array}$} \\
\hline$x(\mathrm{~mm})$ & $y(\mathrm{~mm})$ & & & \\
\hline 100 & 600 & (-42, N.A.) & \multirow{2}{*}{$<1>$} & $(100,560)$ \\
\hline 160 & 600 & $(113$, N.A. $)$ & & $(160,590)$ \\
\hline \multirow{3}{*}{220} & 210 & $(215,71)$ & \multirow{5}{*}{$<2>$} & $(220,200)$ \\
\hline & 450 & (220, N.A. $)$ & & $(220,440)$ \\
\hline & 510 & (196, N.A.) & & $(220,500)$ \\
\hline \multirow{2}{*}{280} & 270 & $(280,394)$ & & $(280,260)$ \\
\hline & 390 & $(283,598)$ & & $(280,390)$ \\
\hline \multirow{2}{*}{340} & 330 & (343, N.A.) & \multirow{4}{*}{$<3>$} & $(340,330)$ \\
\hline & 500 & $(344$, N.A. $)$ & & $(340,500)$ \\
\hline \multirow{2}{*}{400} & 270 & $(402,224)$ & & $(400,250)$ \\
\hline & 390 & $(403,253)$ & & $(410,400)$ \\
\hline \multirow{3}{*}{560} & 210 & $(460,169)$ & \multirow{3}{*}{$<4>$} & $(560,190)$ \\
\hline & 450 & $(467,229)$ & & $(570,450)$ \\
\hline & 510 & $(454,212)$ & & $(570,530)$ \\
\hline
\end{tabular}

It can be seen from Table 6 that when the experimental data are used for TDOA localization, the $\mathrm{x}$-coordinates location are accurate, but the $\mathrm{y}$-coordinates error are relatively large. However, the beamforming localization results are very accurate. Table 7 shows the comparison of the average calculation time between the beamforming method and the joint localization method proposed in this paper under different localization accuracy. Since beamforming scans only a quarter of the steel plate, the localization time of joint localization method is about a quarter of that of beamforming method. For large structures or high-precision localization, the benefits of using the joint localization method are obvious. At the same time, the memory requirements of the computer are reduced.

Table 7. The comparison of the average calculation time between the beamforming method and the joint localization method under different localization accuracy.

\begin{tabular}{ccc}
\hline Localization Accuracy $(\mathbf{m m})$ & Beamforming Method (s) & Joint Localization Method (s) \\
\hline 10 & 2.03 & 0.53 \\
5 & 8.49 & 2.46 \\
2 & 49.16 & 12.08 \\
1 & 193.26 & 50.67 \\
0.5 & 780.19 & 198.16 \\
0.2 & 4887.19 & 1234.80 \\
\hline
\end{tabular}

\section{Conclusions}

This paper proposes a novel joint localization method of AE source based on TDOA method and beamforming method. By using the AE signal from finite element simulation, the performance of TDOA method and beamforming method of four sensors arranged on the plate are studied in detail. Aiming at the advantages and disadvantages of the performance of the two methods, a joint localization method is proposed. The performance of joint localization method is verified by simulation and experiment. Both simulation and experimental results show that the joint localization method not only improves the accuracy of the TDOA method, but also greatly reduces the localization calculation cost of the beamforming method.

Author Contributions: Conceptualization, T.H., X.W. and X.L.; Methodology, X.W. and T.H.; Validation, X.W.; Writing-Original Draft Preparation, X.W.; Writing-Review and Editing, X.W., X.L., T.H., J.T. and Y.S.; Supervision, T.H.; Project Administration, T.H.; Funding Acquisition, T.H. All authors have read and agreed to the published version of the manuscript.

Funding: This work was finically supported by the National Natural Science Foundation of China (Grant No. 51675023), the Key Laboratory of Vibration and Control of Aero-Propulsion System Ministry of 
Education, Northeastern University (VCAME201904) and the Academic Excellence Foundation of BUAA for PhD Students.

Acknowledgments: We thank other members of our lab who provided valuable and constructive suggestions.

Conflicts of Interest: The authors declare no conflict of interest.

\section{References}

1. Wells, R.; Hamstad, M.A.; Mukherjee, A.K. On the origin of the first peak of acoustic emission in 7075 aluminum alloy. J. Mater. Sci. 1983, 18, 1015-1020. [CrossRef]

2. Sause, M.G.R.; Horn, S. Simulation of lamb wave excitation for different elastic properties and acoustic emission source geometries. J. Acoust. Emiss. 2010, 28, 142-154.

3. Lin, L.; Chu, F. HHT-based AE characteristics of natural fatigue cracks in rotating shafts. Mech. Syst. Signal Process. 2012, 26, 181-189. [CrossRef]

4. Wang, Q.; Chu, F. Experimental determination of the rubbing location by means of acoustic emission and wavelet transform. J. Sound Vibr. 2001, 248, 91-103. [CrossRef]

5. Kundu, T.; Das, S.; Martin, S.A.; Jata, K.V. Locating point of impact in anisotropic fiber reinforced composite plates. Ultrasonics 2008, 48, 193-201. [CrossRef] [PubMed]

6. Kundu, T. Acoustic Source Localization. Ultrasonics 2014, 54, 25-38. [CrossRef] [PubMed]

7. Tobias, A. Acoustic-Emission Source Location in Two Dimensions by an Array of Three Sensors. Non-Destr. Test. 1976, 9, 9-12. [CrossRef]

8. Ahadi, M.; Bakhtiar, M.S. Leak Detection in Water-Filled Plastic Pipes through the Application of Tuned Wavelet Transforms to Acoustic Emission Signals. Appl. Acoust. 2010, 71, 634-639. [CrossRef]

9. McLaskey, G.C.; Glaser, S.D.; Grosse, C.U. Beamforming Array Techniques for Acoustic Emission Monitoring of Large Concrete Structures. J. Sound Vib. 2010, 329, 2384-2394. [CrossRef]

10. He, T.; Pan, Q.; Liu, Y.; Liu, X.; Hu, D. Near-field beamforming analysis for acoustic emission source localization. Ultrasonics 2012, 52, 587-592. [CrossRef] [PubMed]

11. Nakatani, H.; Hajzargarbashi, T.; Ito, K.; Kundu, T.; Takeda, N. Impact localization on a cylindrical plate by near-field beamforming analysis. In Sensors and Smart Structures Technologies for Civil, Mechanical, and Aerospace Systems 2012; International Society for Optical Engineering: San Diego, CA, USA, 2012; Volume 8345, p. 83450Y. [CrossRef]

12. Nakatani, H.; Hajzargarbashi, T.; Ito, K.; Kundu, T.; Takeda, N. Locating Point of Impact On An Anisotropic Cylindrical Surface Using Acoustic Beamforming Technique. Key Eng. Mater. 2013, 558, 331-340. [CrossRef]

13. Kundu, T.; Das, S.; Jata, K.V. Point of impact prediction in isotropic and anisotropic plates from the acoustic emission data. J. Acoust. Soc. Am. 2007, 122, 2057-2066. [CrossRef] [PubMed]

14. Kundu, T.; Das, S.; Jata, K.V. Detection of the Point of Impact on a Stiffened Plate by the Acoustic Emission Technique. Smart Mater. Struct. 2009, 18, 35006. [CrossRef]

15. Hajzargerbashi, T.; Kundu, T.; Bland, S. An Improved Algorithm for Detecting Point of Impact in Anisotropic Inhomogeneous Plates. Ultrasonics 2011, 51, 317-324. [CrossRef] [PubMed]

16. Koabaz, M.; Hajzargarbashi, T.; Kundu, T.; Deschamps, M. Locating the Acoustic Source in An Anisotropic Plate. Struct. Health Monit. 2011, 11, 315-323. [CrossRef]

17. Kundu, T. A New Technique for Acoustic Source Localization in an Anisotropic Plate without Knowing Its Material Properties. In Proceedings of the 6th European Workshop on Structural Health Monitoring, Dresden, Germany, 3-6 July 2012; pp. 37-45.

18. Kundu, T.; Nakatani, H.; Takeda, N. Acoustic Source Localization in Anisotropic Plates. Ultrasonics 2012, 52, 740-746. [CrossRef] [PubMed]

19. Nakatani, H.; Kundu, T.; Takeda, N. Improving accuracy of acoustic source localization in anisotropic plates. Ultrasonics 2014, 54, 1776-1788. [CrossRef] [PubMed]

20. He, T.; Xiao, D.; Pan, Q.; Liu, X. Analysis on accuracy improvement of rotor-stator rubbing localization based on acoustic emission beamforming method. Ultrasonics 2014, 54, 318-329. [CrossRef] [PubMed] 
21. Liu, X.; Xiao, D.; Shan, Y.; Pan, Q.; He, T.; Gao, Y. Solder joint failure localization of welded joint based on acoustic emission beamforming. Ultrasonics 2017, 74, 221-232. [CrossRef] [PubMed]

22. He, T.; Tai, J.; Shan, Y.; Wang, X.; Liu, X. A Fast Acoustic Emission Beamforming Localization Method Based on Hilbert Curve. Mech. Syst. Signal Process. 2019, 133, 106291. [CrossRef]

Publisher's Note: MDPI stays neutral with regard to jurisdictional claims in published maps and institutional affiliations.

(C) 2020 by the authors. Licensee MDPI, Basel, Switzerland. This article is an open access article distributed under the terms and conditions of the Creative Commons Attribution (CC BY) license (http://creativecommons.org/licenses/by/4.0/). 